\title{
HD 98618: A STAR CLOSELY RESEMBLING OUR SUN ${ }^{1}$
}

\author{
Jorge Meléndez, ${ }^{2}$ Katie Dodds-Eden, and José A. Robles \\ Research School of Astronomy and Astrophysics, Mount Stromlo Observatory, Cotter Road, Weston Creek, ACT 2611, Australia; \\ jorge@mso.anu.edu.au,katie@mso.anu.edu.au,josan@mso.anu.edu.au \\ Received 2006 February 13; accepted 2006 March 6; published 2006 March 30
}

\begin{abstract}
Despite the observational effort carried out in the last few decades, no perfect solar twin has been found to date. An important milestone was achieved a decade ago by Porto de Mello \& da Silva, who showed that 18 Sco is almost a solar twin. In the present work, we use extremely high resolution $\left(R=10^{5}\right)$, high signal-to-noise ratio Keck HIRES spectra to carry out a differential analysis of 16 solar-twin candidates. We show that HD 98618 is the second-closest solar twin and that the fundamental parameters of both HD 98618 and 18 Sco are very similar (within a few percent) to the host star of our solar system, including the likelihood of hosting a terrestrial planet within their habitable zones. We suggest that these stars should be given top priority in exoplanet and SETI surveys.
\end{abstract}

Subject headings: stars: abundances — stars: activity — stars: atmospheres — stars: fundamental parameters — Sun: fundamental parameters

\section{INTRODUCTION}

The most important question for extrasolar terrestrial planet surveys is which nearby stars should be searched for Earthlike planets (Seager 2003). We are living proof that stars like our Sun can host habitable planets. Thus, stars identical to the Sun (solar twins) may be the best targets for future space missions that will probe Earth-like planets for signs of chemicals that are only produced when life is present.

Since solar twins are very similar (ideally, identical) to the Sun in their fundamental parameters (e.g., temperature, luminosity, metallicity, mass, age), their atmospheric parameters should be very well known, which makes them extremely important for the calibration of fundamental observables in astrophysics, such as the transformation from color to temperature. In particular, we do not yet know accurately the solar $B-V$ color, for which the early literature (1957-1982) shows values from 0.615 to 0.685 (Cayrel de Strobel 1996). Modern values (1994-2006) still show a large spread from a "blue" $(\approx 0.60-0.63$; Straižys \& Valiauga 1994; Taylor 1994; Colina et al. 1996; Sekiguchi \& Fukugita 2000; Ramírez \& Meléndez $2005)$ to a "yellow" ( $\approx 0.64$; Straižys \& Valiauga 1994; Holmberg et al. 2006) to a "red" $(\approx 0.65-0.68$; Gray 1995; Bessell et al. 1998) $(B-V)_{\odot}$ color.

Twins could be useful in the absolute flux calibration of terrestrial and space photometric systems. For example, the recent absolute calibration of the Infrared Array Camera (IRAC) on the Spitzer Space Telescope (Reach et al. 2005) yielded inconsistent (by up to 7\%) results for A and $\mathrm{K}$ stars. With a photometric stability of just over $1 \%$, IRAC is capable of high-precision photometry; however, the accuracy of IRAC data is limited by errors in its absolute flux calibration.

Unusual variations in solar activity seem to be related to climate changes, as may be the case for the "Maunder minimum" (Maunder 1890; Eddy 1976), a period of extremely low

\footnotetext{
${ }^{1}$ The data presented herein were obtained at the W. M. Keck Observatory, which is operated as a scientific partnership among the California Institute of Technology, the University of California, and the National Aeronautics and Space Administration.

${ }^{2}$ Also Department of Astronomy, California Institute of Technology; and affiliated with the Seminario Permanente de Astronomía y Ciencias Espaciales of the Universidad Nacional Mayor de San Marcos, Peru.
}

solar activity that is related to a "little ice age" (Eddy 1976). Thus, it is of the greatest importance to monitor the activity of stars very similar to the Sun.

The identification of stars closely resembling our host star will be of great benefit in answering the long-standing question of the uniqueness of the Sun (e.g., Gustafsson 1998), allowing us to fairly determine whether the Sun is anomalous or not. Cayrel de Strobel (1996) reviewed the efforts made in the first 25 years of the search for solar twins and concluded that none of the large number of stars analyzed is a "perfect good solar twin." Although a perfect solar twin has yet to be found, Porto de Mello \& da Silva (1997) found that 18 Sco (HD 146233) is "the closest ever solar twin." Soubiran \& Triaud (2004) show that 18 Sco is the top solar analog in a sample of about 200 G dwarfs. Very recently, King et al. (2005) analyzed four twin candidates and suggested that HD 143436 is a solar twin as good as 18 Sco.

Here we show that HD 98618 and 18 Sco are the closest solar twins, both with fundamental properties very similar to our Sun.

\section{DATA AND ANALYSIS}

The candidate solar twins were selected based on their blueoptical-red-infrared colors (Ramírez \& Meléndez 2005), chromospheric activity, $v \sin i$, and Hipparcos parallaxes and from previous spectroscopic analysis in the literature (e.g., Valenti \& Fischer 2005). The solar-twin sample is being observed using HIRES at the Keck I Telescope during other observing programs. We are using the upgraded HIRES spectrograph with the highest resolving power $\left(R \approx 10^{5}\right)$, covering $\approx 0.4-0.8 \mu \mathrm{m}$ on the "blue," "green," and "red" chips.

So far, 16 candidates (listed in Table 1) have been observed in 2005 and 2006. Each star was observed for 1-3 minutes in order to achieve a signal-to-noise ratio of at least $\approx 300$ per resolution element (or about 200 pixel $^{-1}$ ) at $671 \mathrm{~nm}$. For most stars, we fulfilled this requirement. During each run, a solar reflected spectrum was obtained by observing either Ceres or Vesta. The data were reduced employing the latest version of MAKEE (T. Barlow 2005, private communication). Further data reductions (Doppler correction and continuum normali- 
TABLE 1

Slope $d\left(\delta d_{r}\right) / d\left(\chi_{\text {exc }}\right)$ FOR Fe I ANd Relative Differences IN LINE DEPTH $\left\langle\delta d_{r}\right\rangle$ FOR Fe I, V I, AND Ca I

\begin{tabular}{|c|c|c|c|c|}
\hline Star & Slope & $\mathrm{Fe} I$ & $\mathrm{~V}_{\mathrm{I}}$ & $\mathrm{Ca} \mathrm{I}$ \\
\hline ID 9986 . & -0.0120 & -0.042 & -0.100 & -0.019 \\
\hline D 32963 & & & & \\
\hline D 33636 & 0.0 & & & 0.116 \\
\hline HD 45184 & -0.00 & & & 0.004 \\
\hline HD $56124 \ldots \ldots$ & & & & 0.010 \\
\hline 334 & -0.0 & & & -0.027 \\
\hline HD 7 & -0.0 & & & 0.021 \\
\hline HD 718 & & & & 0.045 \\
\hline HD 98618 & -0.003 & -0.0 & -0.0 & 0.002 \\
\hline & & & -0 . & -0.070 \\
\hline HD & -0.0 & & 0.0 & 0.011 \\
\hline HD 146233 & -0.0007 & -0.001 & 0.024 & -0.004 \\
\hline HD 147044 & 0.0040 & & 0.270 & 0.055 \\
\hline HD 159222 & -0.0160 & -0. & 0.000 & 0.013 \\
\hline & & & -0.213 & -0.053 \\
\hline HD 187123 . & -0.0120 & -0.081 & -0.153 & -0.052 \\
\hline
\end{tabular}

zation) were performed with IRAF. Sample spectra of the Sun, 18 Sco, HD 98618, and HD 143436 are shown in Figure 1.

Orders falling on the "green" chip, which in our setup covers $\approx 0.53-0.69 \mu \mathrm{m}$, were scrutinized for the best lines available for analysis. We selected lines of moderate strength (typically with line depths of $0.2-0.6$, but weaker and stronger lines were also included), unblended, relatively symmetric, and free of telluric contamination. Lines too close to the edges of the orders were discarded because of the greater uncertainty in fitting the continuum in these regions.

We employed two basic criteria to determine how close a star is to the Sun. The first criterion assessed the similarity between spectral lines in the sample star relative to the Sun for different chemical elements. For each atomic line, we determined the relative difference in line depth $\delta d_{r}$ employing the 3 closest pixels to the line center. We critically selected 206 lines of 10 ions, with the largest proportion (70) due to $\mathrm{Fe} \mathrm{I}$. A median $\left\langle\delta d_{r}\right\rangle$ was determined for each ion, and errors were estimated. A perfect solar twin should have $\left\langle\delta d_{r}\right\rangle=0$ for lines of all the chemical elements, and although none was found, 18 Sco and HD 98618 showed the $\left\langle\delta d_{r}\right\rangle$ values closest to zero (i.e., solar) among the different chemical elements analyzed.
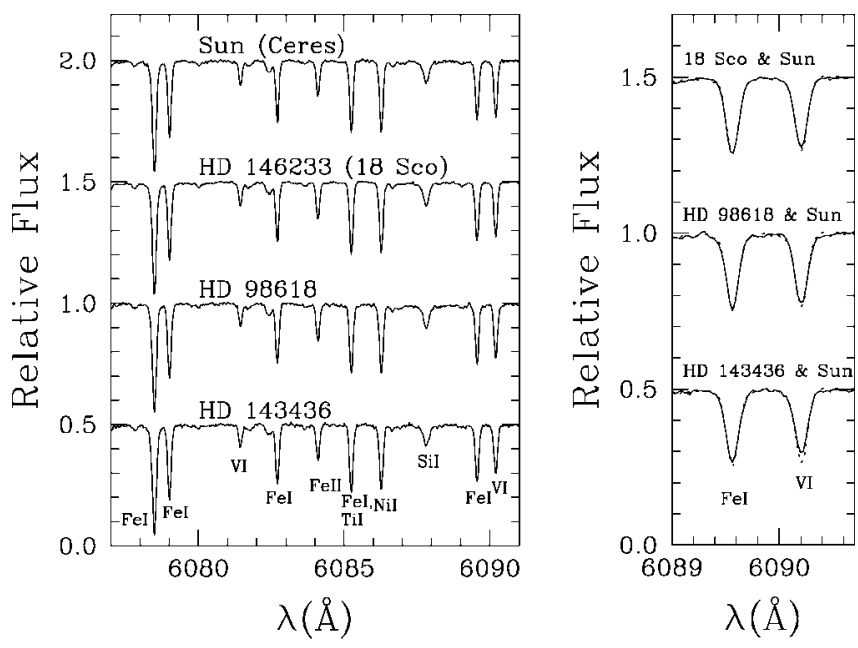

FIG. 1.-Left, sample spectra of the Sun and the candidate solar twins 18 Sco, HD 98618, and HD 143436; right, comparison of the solar-twin candidates (solid lines) with the Sun (dotted line) around $6090 \AA$ A. Since V I is very sensitive to $T_{\text {eff }}$, the weaker V I line in HD 143436 suggests that this star has a $T_{\text {eff }}$ higher than solar, while both $18 \mathrm{Sco}$ and HD 98618 are very similar to the Sun.

The second criterion allowed us to identify how close a star is in temperature to the Sun. The principle behind this is the excitation equilibrium of $\mathrm{Fe}$ I lines, which are very sensitive to effective temperature. For a star identical to the Sun, the slope of a plot of $\delta d_{r}(\mathrm{Fe}$ I $)$ versus excitation potential $\chi_{\text {exc }}$ should be zero. In addition to the $\mathrm{Fe} \mathrm{I}$ lines used in our first criterion, we selected four lines around $525 \mathrm{~nm}$ from the "blue" chip, three of them close to $\chi_{\mathrm{exc}}=0 \mathrm{eV}$ and hence allowing a better determination of the $d\left(\delta d_{r}\right) / d \chi_{\text {exc }}$ slope.

The results of the two criteria are shown in Table 1 and Figure 2, where it can be seen that 18 Sco is the best solar twin out of our candidates, with HD 98618 also appearing very close to the Sun. Figure 2 also shows that, according to our criteria, HD 143436 is no match for 18 Sco, in contrast to the suggestion (King et al. 2005) that they may be equivalent. In addition, we estimated the relative depths of the Li feature at $6708 \AA$ A H 98618 and 18 Sco have similar Li profiles, which
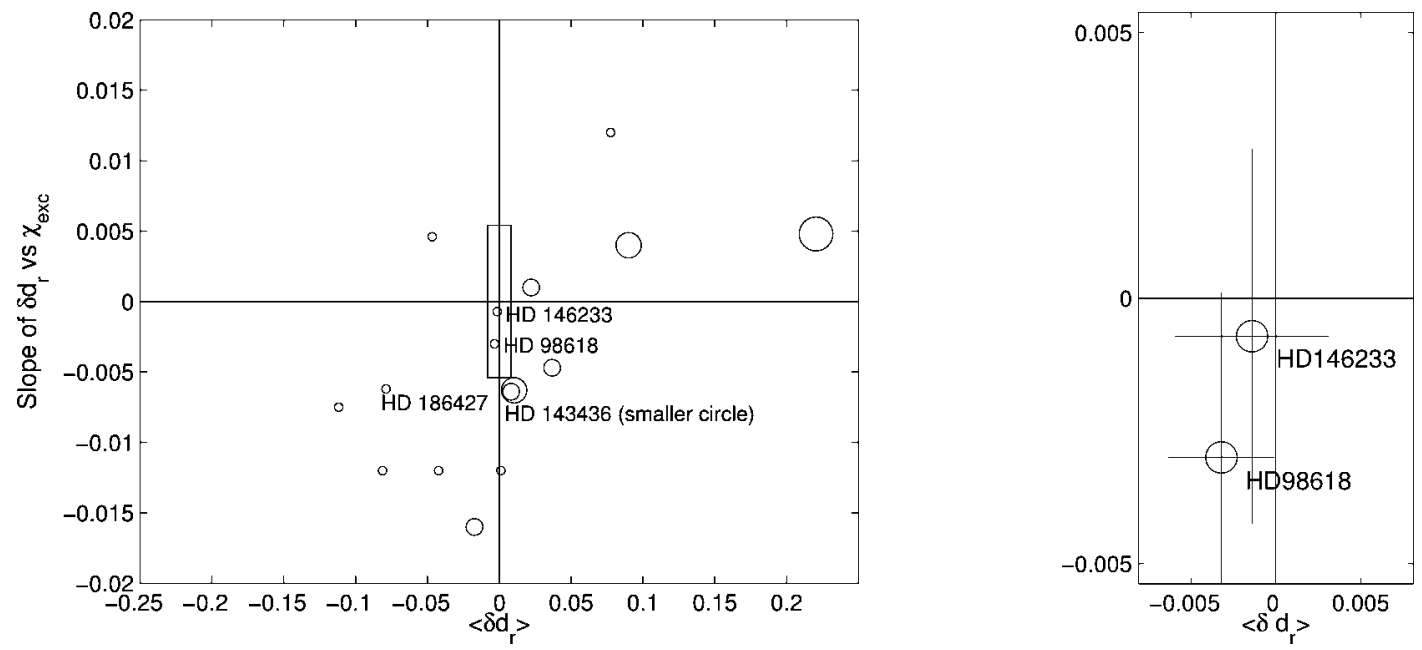

FIG. 2.-Slope $d\left(\delta d_{r}\right) / d \chi_{\text {exc }}$ vs. the median relative difference in line depth $\left\langle\delta d_{r}\right\rangle$ for Fe I lines (§ 2). A perfect solar twin should be at $(0,0)$. The size of the circles is proportional to the $\mathrm{Li}$ I line depth at $6708 \AA$, with the smallest circles representing closer agreement to the solar Li I profile. Left, the whole sample, with the inner rectangle representing the typical $\pm 1 \sigma$ error bars; right, a zoom to the inner rectangle of the left panel. The two best solar-twin candidates, 18 Sco (HD 146233) and HD 98618, are labeled; HD 143436 (King et al. 2005) and HD 186427 (one of the most promising twin candidates in the early literature) are shown as well. 
TABLE 2

Fundamental Parameters

\begin{tabular}{|c|c|c|}
\hline Parameter (Star - Sun) & $18 \mathrm{Sco}$ & HD 98618 \\
\hline$\overline{\Delta v_{t}\left(\mathrm{~km} \mathrm{~s}^{-1}\right)}$ & $+0.08 \pm 0.15$ & $+0.09 \pm 0.15$ \\
\hline$\Delta T_{\text {eff }}(\mathrm{K}) \ldots$ & $+40 \pm 30$ & $+66 \pm 30$ \\
\hline$\Delta \log g_{\text {spec }}(\mathrm{dex})$ & $+0.01 \pm 0.04$ & $+0.01 \pm 0.04$ \\
\hline$\Delta \log g_{\text {Hip }}($ dex $) \ldots .$. & $+0.01 \pm 0.02$ & $+0.01 \pm 0.03$ \\
\hline$\Delta \log g_{\text {adopted }}(\mathrm{dex})$ & $+0.01 \pm 0.02$ & $+0.01 \pm 0.03$ \\
\hline$\Delta L_{\text {spec }}(L$. & $+0.02 \pm 0.06$ & $+0.04 \pm 0.06$ \\
\hline$\Delta L_{\text {Hip }}^{\text {spec }}\left(L_{\odot}\right) \ldots$. & $+0.03 \pm 0.03$ & $+0.08 \pm 0.07$ \\
\hline$\Delta L_{\text {adopted }}\left(L_{\odot}\right)$ & $+0.03 \pm 0.02$ & $+0.06 \pm 0.05$ \\
\hline$[\mathrm{Fe} / \mathrm{H}](\mathrm{dex}) \ldots \ldots$ & $+0.02 \pm 0.03$ & $+0.05 \pm 0.03$ \\
\hline$[\mathrm{O} / \mathrm{H}](\mathrm{dex}) \ldots \ldots$ & $-0.03 \pm 0.05$ & $0.00 \pm 0.04$ \\
\hline$[\mathrm{Li} / \mathrm{H}](\mathrm{dex})$ & $+0.53 \pm 0.09$ & $+0.47 \pm 0.09$ \\
\hline$\Delta \operatorname{mass}\left(M_{\odot}\right) \ldots \ldots$ & $+0.02 \pm 0.03$ & $+0.02 \pm 0.03$ \\
\hline$\Delta$ age $_{\text {isochro }}(\mathrm{Gyr}) \ldots$ & $-0.8 \pm 1.5$ & $-1.1 \pm 1.5$ \\
\hline$\Delta$ age $_{\text {chromos }}(\mathrm{Gyr})$ & $-0.3^{\mathrm{a}}$ & $+0.7^{\mathrm{a}}$ \\
\hline$\Delta$ age $_{\text {rotation }}(\mathrm{Gyr}) \ldots$ & -1.1 & -0.4 \\
\hline$\Delta$ age $_{\text {dopted }}(\mathrm{Gyr})$ & $-0.7 \pm 0.4$ & $-0.3 \pm 0.9$ \\
\hline$\Delta$ rotation period (days) $\ldots \ldots$ & $-2.5^{\mathrm{b}},-1^{\mathrm{a}}$ & $-1^{\mathrm{a}}$ \\
\hline$\Delta \log R_{\mathrm{HK}}^{\prime}(\operatorname{dex}) \ldots \ldots \ldots \ldots \ldots$ & $0.0^{\mathrm{a}}$ & $-0.05^{\mathrm{a}}$ \\
\hline$\Delta M_{V}(\mathrm{mag}) \ldots \ldots \ldots \ldots \ldots \ldots$ & $-0.04 \pm 0.04$ & $-0.09 \pm 0.07$ \\
\hline$B-V \ldots \ldots \ldots \ldots \ldots \ldots \ldots \ldots \ldots \ldots \ldots \ldots \ldots$ & 0.65 & 0.64 \\
\hline Distance $(\mathrm{pc}) \ldots \ldots \ldots \ldots \ldots$ & 14.0 & 38.7 \\
\hline
\end{tabular}

are both deeper than solar yet much closer to the Sun's than the Li profile of HD 143436. Hence, also considering the Li abundance, HD 143436 is not a good solar twin. The spectroscopic $\log g(=4.28)$ determined by King et al. (2005) is considerably lower than the trigonometric $\log g$ from Hipparcos parallaxes $\left(\approx 4.46\right.$, using the $T_{\text {eff }}$ and mass given by them), which suggests errors in their analysis. Since the spectroscopic $\log g$ is too low, the spectroscopic $T_{\text {eff }}$ given by King et al. is probably also underestimated.

It is important to note that our analysis is strictly differential and independent of model atmospheres, with both the sample and reflected solar spectrum (Vesta or Ceres) obtained employing the same HIRES setting and during the same run and the data reduction and continuum normalization performed in the same way. On the other hand, King et al. (2005) used a lunar spectrum obtained several years earlier, and their observations are of lower resolving power $(R \approx 45,000)$. The set of lines used is also different; while King et al. cover 647$675 \mathrm{~nm}$, our Fe I lines cover 522-675 nm. Note that part of the region used by them has weak telluric contamination.

\section{DETAILED ANALYSIS OF 18 SCORPII AND HD 98618}

The top twin candidates, 18 Sco and HD 98618, were analyzed in more detail to quantify their likeness to the Sun by performing a differential spectroscopic equilibrium analysis with respect to the solar spectrum. The close proximity of our candidates to the Sun warrants reliable relative chemical and physical parameters.

The equivalent widths of $39 \mathrm{Fe}$ I and $14 \mathrm{Fe}$ II lines with clean profiles and well-defined continuum regions were mea- sured in the region 439-675 $\mathrm{nm}$. The $g$-values of $\mathrm{Fe} \mathrm{I}$ and $\mathrm{Fe}$ II lines were taken from the Oxford (e.g., Blackwell et al. 1995) and Hannover groups (e.g., Bard \& Kock 1994) and from Meléndez et al. (2006), respectively. Oxygen and lithium abundances were obtained from the $\mathrm{O}$ I triplet at $777 \mathrm{~nm}$ and the $\mathrm{Li}$ I profile at $670.7 \mathrm{~nm}$. The interaction-broadening constants $C_{6}$ were obtained from the collision-broadening cross sections given by Barklem et al. (2000) and Barklem \& Aspelund-Johansson (2005).

The LTE calculations were performed with the 2002 version of MOOG (Sneden 1973), employing Kurucz overshooting model atmospheres (Castelli et al. 1997). The microturbulence $v_{t}$ was determined by requiring no dependence of the iron abundance from $\mathrm{Fe}$ I lines $\left(A_{\mathrm{Fe}}\right)$ against reduced equivalent width $\left(W_{\lambda} / \lambda\right)$.

The zero point of the excitation and ionization equilibrium was defined by the analysis of the solar $\mathrm{Fe} \mathrm{I}$ and $\mathrm{Fe}$ II lines: $\left(d A_{\mathrm{Fe} \mathrm{I}} / d \chi_{\text {exc }}\right)_{\odot}$ and $\left(\Delta_{\mathrm{Fe} \mathrm{II}-\mathrm{FeI}}\right)_{\odot}\left(=A_{\mathrm{Fe} \mathrm{II}}-A_{\mathrm{FeI}}\right)$. For the Sun we adopted the atmospheric parameters $\left(T_{\text {eff }}, \log g,[\mathrm{Fe} / \mathrm{H}]\right)=$ $(5777 \mathrm{~K}, 4.44,0.0)$, which was also adopted as a first guess for the twin candidates. Then the parameters of the sample stars were iteratively changed in $\left(\Delta T_{\text {eff }}, \Delta \log g, \Delta[\mathrm{Fe} / \mathrm{H}]\right)$ until we simultaneously achieved relative excitation and ionization equilibrium:

$$
\begin{aligned}
\left(d A_{\mathrm{Fe}} / d \chi_{\mathrm{exc}}\right)_{*} & =\left(d A_{\mathrm{Fe} \mathrm{I}} / d \chi_{\mathrm{exc}}\right)_{\odot}, \\
\left(\Delta_{\mathrm{Fe} I}-\mathrm{Fe} \mathrm{I}\right. & )_{*}=\left(\Delta_{\mathrm{Fe} \mathrm{II}-\mathrm{Fe} \mathrm{I}}\right)_{\odot} .
\end{aligned}
$$

The stellar parameters so determined were used to obtain stellar masses and ages from $\mathrm{Y}^{2}$ isochrones (Demarque et al. 2004). Once the mass was estimated, a spectroscopic luminosity was obtained from $\log \left(L / L_{\odot}\right)=\log \left(M / M_{\odot}\right)-\log \left(g / g_{\odot}\right)+$ $4 \log \left(T_{\text {eff }} / T_{\text {eff, } \odot}\right)$.

We employed Hipparcos parallaxes to check both the surface gravities and luminosities obtained in the present spectroscopic analysis. The trigonometric gravity ( $\log g_{\mathrm{Hip}}$ ) and stellar luminosity $\left(L_{\mathrm{Hip}}\right)$ were calculated employing Hipparcos parallaxes, $V, M_{\mathrm{bol}, \odot}=4.736$ (Bessell et al. 1998), and the bolometric correction

$$
\mathrm{BC}=-1.6240+4.5066 \theta-3.12936 \theta^{2} \quad(\sigma=0.02 \mathrm{mag}),
$$

where $\theta=(5040 \mathrm{~K}) / T_{\text {eff }}$. BC was obtained from a fit to solarmetallicity dwarfs from Alonso et al. (1995) and Blackwell \& Lynas-Gray (1998); the relation above is valid from about 4200 to $7000 \mathrm{~K}$.

The spectroscopic and Hipparcos-based results are shown in Table 2, where one can see the excellent agreement in both $\log g$ and luminosity, which lends confidence to the results obtained in the present work. A comparison with previous differential spectroscopic analyses is shown in Table 3. All agree that the atmospheric parameters of 18 Sco and HD 98618 are very similar to the Sun, especially in $T_{\text {eff }}(1.1 \%$ or better). The differences in $\log g$ and $[\mathrm{Fe} / \mathrm{H}]$ are just within a few times

TABLE 3

Comparison of Relative Spectroscopic Parameters $\Delta\left(T_{\text {eff }} / \log g /[\mathrm{Fe} / \mathrm{H}]\right)$

\begin{tabular}{lcc}
\hline \hline Source & $\begin{array}{c}18 \text { Sco } \\
(\text { K/dex/dex })\end{array}$ & $\begin{array}{c}\text { HD } 98618 \\
(\text { K/dex } / \text { dex })\end{array}$ \\
\hline This work $\ldots \ldots \ldots \ldots \ldots \ldots \ldots \ldots \ldots$ & $+40 /+0.01 /+0.02$ & $+66 /+0.01 /+0.05$ \\
Porto de Mello \& da Silva 1997 $\ldots \ldots$. & $+12 /+0.05 /+0.05$ & $\ldots$ \\
Luck \& Heiter 2005 ............... & $+58 /+0.03 /+0.03$ & $\ldots$ \\
Valenti \& Fischer 2005 $\ldots \ldots \ldots \ldots \ldots$. & $+14 /-0.03 /+0.03$ & $+35 /-0.02 /+0.03$ \\
\hline
\end{tabular}


0.01 dex, almost the same as the typical uncertainties. Although within the uncertainties all these works agree with each other, the trigonometric gravities from Hipparcos parallaxes (Table 2) favor our results.

Rotation periods and stellar ages can be obtained from measurements of stellar activity. Table 2 shows the relative chromospheric activity indices $\left(\log R_{\mathrm{HK}}^{\prime}\right)$, chromospheric ages, and rotation periods estimated by Wright et al. (2004). A better rotation period for HD 146233 is given by Frick et al. (2004), who analyzed time series of chromospheric activity.

The rotation periods can be used to estimate a rotational age (Barry 1988). The relation between age $t$ and rotation period $P$ also depends on mass $\left[\log P=a \log t+b \log \left(M / M_{\odot}\right)+c\right.$; Kawaler 1989), but since our stars have about $1 M_{\odot}$, this relation simplifies to $P \propto t^{a}$. Barry (1988) finds that $a \approx 1 / e$, and using a mean solar rotation period (equator to latitude $20^{\circ}$ ) $P_{\odot} \approx 25.3$ days (Howard 1984) and a solar main-sequence age of $t_{\odot}=4.51$ Gyr (Sackmann et al. 1993), we find $t=$ $(P / 14.5)^{e}$, where $P$ is in days and $t$ is in Gyr. Using this relation, we estimated rotational ages (Table 2 ) adopting $P$ from Frick et al. (2004) and Wright et al. (2004) for 18 Sco and HD 98618, respectively.

\section{DISCUSSION AND CONCLUSIONS}

As shown in Table 2, 18 Sco and HD 98618 are both very similar to the Sun, with the surface gravity being just $0.01 \mathrm{dex}$ higher than solar, the mass above solar by $2 \%, T_{\text {eff }}$ hotter by $0.7 \%$ and $1.1 \%$, metallicity higher by 0.02 and 0.05 dex, and luminosity about $3 \%$ and $6 \%$ higher, respectively. Both stars belong to the disk population and, as the Sun, are orbiting the center of our Galaxy at $\approx 8$ kpc (Nordström et al. 2004).
Both twin candidates seem to be rotating slightly faster than the Sun and are about $10 \%$ younger than the Sun. The higher $\mathrm{Li}$ abundance in $18 \mathrm{Sco}$ and HD 98618 is perhaps due to different amounts of pre-main-sequence depletion (see, e.g., Ventura et al. 1998). Since 18 Sco and HD 98618 are about 4 Gyr old, hypothetical terrestrial planets may have had enough time to develop some kind of complex life, assuming the timescale for formation of complex life is similar to Earth's. Thus, we encourage SETI programs to give top priority to these stars.

Thanks to the long-term effort of the California and Carnegie Planet Search Project (see, e.g., Marcy et al. 2005), we know that there are not any hot Jupiters around 18 Sco or HD 98618. According to Marcy et al. (2005) "among the nearest FGK main-sequence stars $(d<40 \mathrm{pc})$ the yet undiscovered giant planets typically reside beyond $1 \mathrm{AU}$ because giant planets within 1 AU have already been found." Furthermore, Marcy et al. estimate that undetected Jupiter-mass planets orbiting nearby FGK stars $(<30 \mathrm{pc})$ may reside in orbits beyond 3 AU or have masses less than $M_{\text {Jup }}$. Since the distance at which an Earth-size planet would receive the same amount of light from its star as Earth receives from the Sun is $\left(L / L_{\odot}\right)^{1 / 2} \mathrm{AU}$ and the luminosities of the twin candidates are very close to solar, the habitable zones of 18 Sco and HD 98618 should be very close to $1 \mathrm{AU}$. If further radial velocity observations show that the inner region around 18 Sco and HD 98618 is free of giant planets, then these stars have the potential to host terrestrial planets around their habitable zones. We encourage long-term radial velocity monitoring of these stars, which could reveal within a few years how far out gaseous giant planets, if any, reside within the exoplanetary systems these stars may host.

\section{REFERENCES}

Alonso, A., Arribas, S., \& Martínez-Roger, C. 1995, A\&A, 297, 197

Bard, A., \& Kock, M. 1994, A\&A, 282, 1014

Barklem, P. S. \& Aspelund-Johansson, J. 2005, A\&A, 435, 373

Barklem, P. S., Piskunov, N. \& O'Mara, B. J. 2000, A\&AS, 142, 467

Barry, D. C. 1988, ApJ, 334, 436

Bessell, M. S., Castelli, F., \& Plez, B. 1998, A\&A, 333, 231 (erratum 337, 321)

Blackwell, D. E.\& Lynas-Gray, A. E. 1998, A\&AS, 129, 505

Blackwell, D. E., Lynas-Gray, A. E., \& Smith, G. 1995, A\&A, 296, 217

Castelli, F., Gratton, R. G., \& Kurucz, R. L. 1997, A\&A, 318, 841 (erratum $324,432)$

Cayrel de Strobel, G. 1996, A\&A Rev., 7, 243

Colina, L., Bohlin, R. C., \& Castelli, F. 1996, AJ, 112, 307

Demarque, P., Woo, J.-H., Kim, Y.-C., Yi, S. K. 2004, ApJS, 155, 667

Eddy, J. A. 1976, Science, 192, 1189

Frick, P., Soon, W., Popova, E., \& Baliunas, S. 2004, NewA, 9, 599

Gray, D. F. 1995, PASP, 107, 120

Gustafsson, B. 1998, Space Sci. Rev., 85, 419

Holmberg, J., Flynn, C., \& Portinari, L. 2006, MNRAS, 367, 449

Howard, R. 1984, ARA\&A, 22, 131

Kawaler, S. D. 1989, ApJ, 343, L65

King, J. R., Boesgaard, A. M., \& Schuler, S. C. 2005, AJ, 130, 2318
Luck, R. E., \& Heiter, U. 2005, AJ, 129, 1063

Marcy, G. W., Butler, R. P., Vogt, S. S., Fischer, D. A., Henry, G. W., Laughlin, G., Wright, J. T., \& Johnson, J. A. 2005, ApJ, 619, 570

Maunder, E. W. 1890, MNRAS, 50, 251

Meléndez, J., Shchukina, N. G., Vasiljeva, I. E. \& Ramírez, I. 2006, ApJ, in press (astro-ph/0601256)

Nordström, B., et al. 2004, A\&A, 418, 989

Porto de Mello, G. F., \& da Silva, L. 1997, ApJ, 482, L89

Ramírez, I., \& Meléndez, J. 2005, ApJ, 626, 465

Reach, W. T., et al. 2005, PASP, 117, 978

Sackmann, I.-J., Boothroyd, A. I., \& Kraemer, K. E. 1993, ApJ, 418, 457

Seager, S. 2003, Earth Planet. Sci. Lett., 208, 113

Sekiguchi, M., \& Fukugita, M. 2000, AJ, 120, 1072

Sneden, C. 1973, Ph.D. thesis, Univ. Texas

Soubiran, C., \& Triaud, A. 2004, A\&A, 418, 1089

Straižys, V., \& Valiauga, G. 1994, Baltic Astron., 3, 282

Taylor, B. J. 1994, PASP, 106, 444

Valenti, J. A., \& Fischer, D. A. 2005, ApJS, 159, 141

Ventura, P., Zeppieri, A., Mazzitelli, I., \& D’Antona, F. 1998, A\&A, 331, 1011

Wright, J. T., Marcy, G. W., Butler, R. P., \& Vogt, S. S. 2004, ApJS, 152, 261

Note added in proof.-After this Letter was accepted for publication, M. C. Turnbull brought it to our attention that HD 98618 is included in the Catalog of Nearby Habitable Stellar Systems (M. C. Turnbull \& J. C. Tarter, ApJS, 145, 181; ApJS, 149, 423 [2003]) for SETI searches with the Allen Telescope Array. It is ranked No. 106 in terms of its location on the H-R diagram, and considering its distance merits its final ranking of No. 504. The present work suggests that HD 98618 should be given a higher ranking. 